\title{
Herramientas de autor que apoyan la creación de contenido web con propósito educativo en Colombia
}

David Andrés Vargas-Agreda ${ }^{1}$

Universidad Pontificia Bolivariana

david.vargasa@upb.edu.co

ORCID: https://orcid.org/0000-0003-3691-7411

Silvia Baldiris ${ }^{2}$

Universidad Internacional de la Rioja

silvia.baldiris@unir.net

ORCID: https://orcid.org/0000-0002-6818-3427

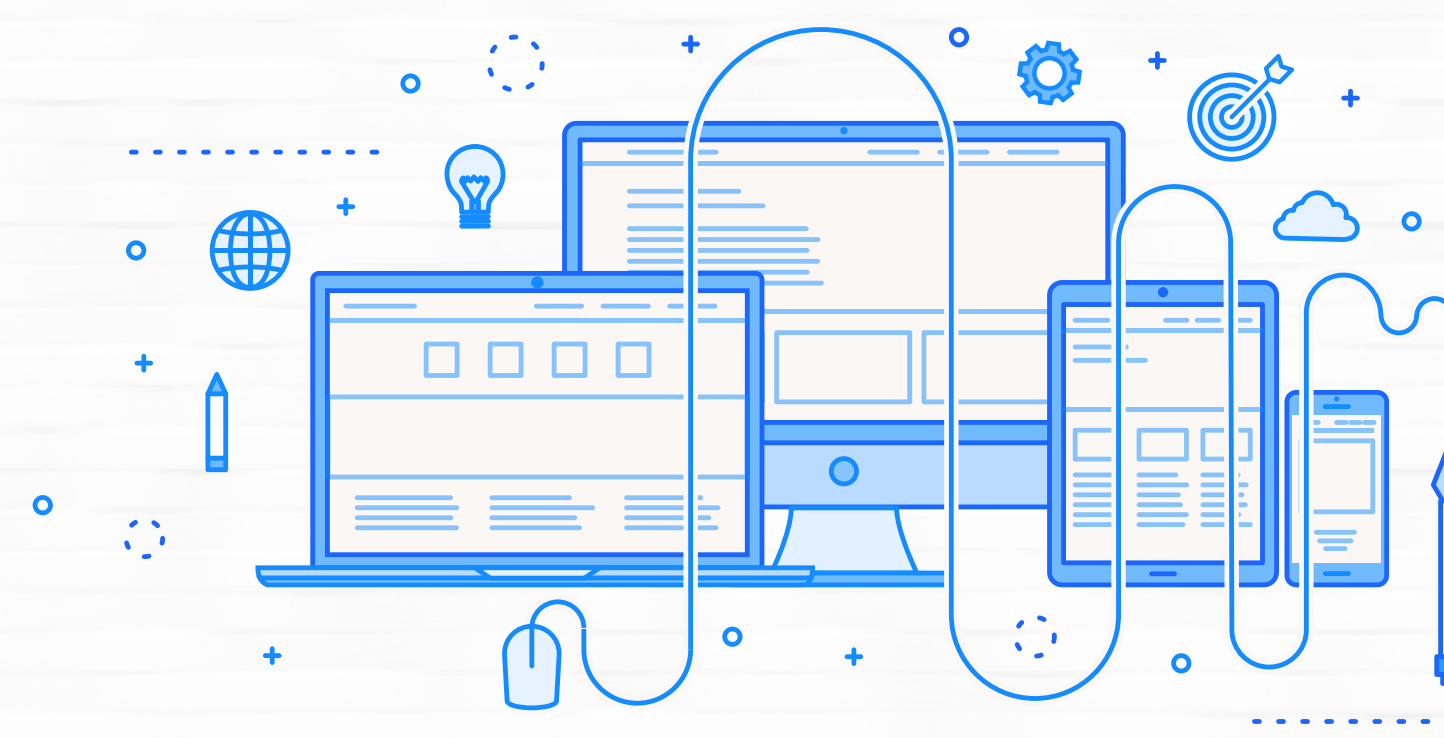

DOI: https://doi.org/10.21158/2357514x.v7.n1.2019.2317

Cómo citar este artículo: Vargas-Agreda, D. A.; Baldiris, S. (2019). Herramientas de autor que apoyan la creación de contenido web con propósito educativo en Colombia. Revista Virtu@Imente, 7(1), 11-28. DOI: https://doi.org/10.21158/2357514x.v7.n1.2019.2317

Fecha de recepción: 11 de enero de 2019

Fecha de aprobación: 19 de marzo de 2019

1 Ingeniero en Sistemas por la Pontificia Universidad Católica del Ecuador Sede Ibarra (PUCESI). Magíster en Tecnologías de la Información y la Comunicación por la Universidad Pontificia Bolivariana (UPB) en Medellín, Colombia.

2 Ingeniera de Sistemas e Industrial de la Universidad Industrial de Santander, Colombia. Magíster en Informática Industrial y Automática de la Universidad de Girona y Doctora en Tecnología de la

Universitat de Girona. Desarrolló un postdoctorado en la Universidad de Athabasca en Edmonton (Canadá) en el área de Métodos de Investigación Aplicados a la Educación con apoyo de las TIC. 


\section{RESUMEN}

El avance de las Tecnologías de la Información y Comunicación (TIC) ha conllevado al uso de un número creciente de herramientas de autor en la educación, por ello, en este estudio, con base en una revisión bibliográfica en repositorios académicos digitales y la posterior aplicación de una encuesta en línea a un grupo de docentes colombianos, se logró identificar las variables socio demográficas y competencias digitales de los encuestados y las herramientas de autor más usadas para la creación de contenido educativo.

Dentro del estudio se evidenció que si bien los docentes manifestaron tener competencias digitales para el uso del computador e internet, así como estar de acuerdo con la importancia del uso de las TIC para apuntalar la transformación, innovación y mejora de los métodos tradicionales de enseñanza y aprendizaje, esto no ha sido adoptado por la mayoría de ellos como didáctica de uso permanente en las aulas, lo que se demuestra gracias a la frecuencia ocasional con la que diseñan recursos didácticos mediados por TIC como herramienta en su práctica docente.

De otra parte se encontró que se hace necesaria una sensibilización para los docentes, con el objetivo de promover la creación de material educativo mediado por TIC que considere los lineamientos de accesibilidad web, incluyendo así a una mayoría de alumnado, con diferentes necesidades y estilos de aprendizaje.

Palabras clave: herramientas de autor; tecnología en la educación; competencias digitales; métodos de enseñanza; métodos de aprendizaje, herramientas educativas; accesibilidad en la educación; recursos didácticos; innovación en educación. 


\section{Author tools that support the creation of web content for educational purposes in Colombia}

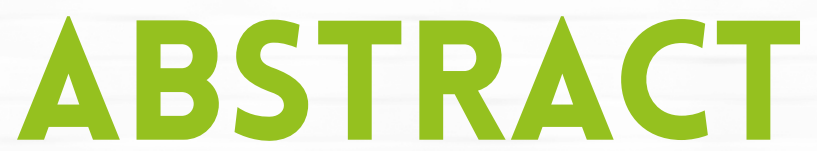

The development of the Information and Communication Technologies (ICT) has led to the use of an increasing number of authoring tools in education, therefore, this study, which is based on a bibliographic review in digital academic repositories and the subsequent application of an online survey to a group of Colombian teachers, makes it possible to identify the socio-demographic variables and digital skills of the respondents and the most used authoring tools for the creation of educational content.

Within the study, it became apparent that although the teachers stated that they had digital competences for the use of computers and the Internet, and that they agreed with the importance of the use of the ICT to underpin the transformation, innovation, and improvement of traditional teaching and learning methods, this has not been adopted by most of them as didactics for permanent use in the classroom, which is demonstrated by the occasional frequency with which they design ICT-mediated teaching resources as a tool in their teaching practice.

On the other hand, it was found that sensitization is necessary for teachers, aiming at promoting the creation of ICT-mediated educational material that considers the web accessibility guidelines, including a majority of students, with different learning needs and styles.

Keywords: authoring tools; technology in education; digital skills; teaching methods; learning methods; educational tools; accessibility in education; didactic resources; innovation in education. 


\section{Ferramentas de autor que apoiam a criação de conteúdo da web para fins educacionais na Colômbia}

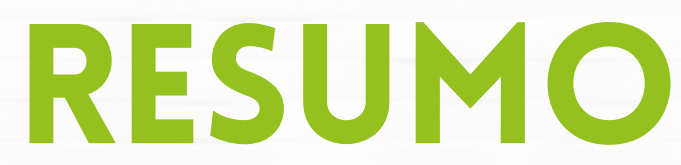

O avanço das Tecnologias da Informação e Comunicação (TIC) levou ao uso de um número crescente de ferramentas de autor na educação, portanto, neste estudo, com base em uma revisão bibliográfica em repositórios acadêmicos digitais e na aplicação subsequente de uma pesquisa on-line a um grupo de professores colombianos, foi possível identificar as variáveis sociodemográficas e habilidades digitais dos entrevistados e as ferramentas de autor mais utilizadas para a criação de conteúdo educacional.

No estudo, evidenciou-se que, embora os professores tenham declarado possuir competências digitais para o uso do computador e da Internet, bem como concordando com a importância do uso das TIC para sustentar a transformação, inovação e aprimoramento dos métodos e métodos tradicionais de ensino e aprendizagem, isso não foi adotado pela maioria deles como didática para uso permanente em sala de aula, o que é demonstrado pela frequência ocasional com a qual eles projetam recursos de ensino mediados pelas TIC como uma ferramenta em sua prática de ensino.

Por outro lado, verificou-se que a sensibilização é necessária para os professores, com o objetivo de promover a criação de material educacional mediado pelas TIC que considere as diretrizes de acessibilidade da web, incluindo a maioria dos estudantes, com diferentes necessidades e estilos de ensino.

Palavras-chave: ferramentas de autor; tecnologia na educação; habilidades digitais; métodos de ensino; métodos de aprendizagem, ferramentas educacionais; acessibilidade na educação; recursos didáticos; Inovação na educação. 


\section{Outils d'aide à la création de contenu internet à des fins éducatives en Colombie}

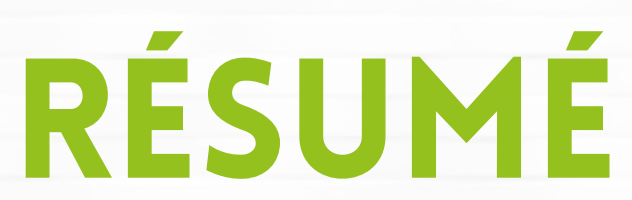

L'avancée des technologies de l'information et de la communication (TIC) a conduit à l'utilisation d'un nombre croissant d'outils éducatifs. Cette étude s'appuie sur une analyse bibliographique des référentiels numériques universitaires et de leur application ultérieure. C'est à partir d'une enquête en ligne auprès d'un groupe d'enseignants colombiens qu'il a été possible d'identifier les variables sociodémographiques et les compétences numériques des personnes interrogées ainsi que les outils les plus utilisés pour la création de contenu éducatif.

Dans le cadre de cette étude, il a été possible de démontrer que les enseignants disposant des compétences numériques pour l'utilisation d'ordinateurs et d'Internet et reconnaissant par ailleurs l'importance de I'utilisation des TIC pour la transformation, l'innovation et le perfectionnement des méthodes d'enseignement traditionnelles et d'apprentissage n'adoptent pas suffisamment cette approche didactique durant leurs classes, comme le montre la fréquence toute relative avec laquelle ils conçoivent des ressources pédagogiques au moyen des TIC.

D'autre part, il est nécessaire de sensibiliser les enseignants à la promotion et la création de matériel pédagogique utilisant les TIC compte tenu des lignes directrices relatives à l'accessibilité d'internet utilisé par une majorité d'élèves ayant des besoins et des styles d'apprentissage différents.

Mots-cléfs: outils de création; technologie et éducation; compétences numériques; méthodes d'enseignement; méthodes d'apprentissage, outils pédagogiques; accessibilité de l'éducation; ressources pédagogiques; innovation en éducation. 


\section{Introducción}

El surgimiento y crecimiento exponencial de la World Wide Web -WWW o simplemente la web- ha permitido el desarrollo de un creciente número de herramientas de autor para la creación de contenidos para apoyar los procesos de enseñanza/aprendizaje, tanto para usuarios expertos como para aquellos sin conocimientos en un lenguaje de programación. Actualmente, una gran cantidad de estas herramientas de software - tanto de pago como gratuitasestán a disposición de los educadores a través del internet; así permiten tanto la creación de entornos virtuales de enseñanza/aprendizaje (Crosetti, 2008) como también de material educativo en formato digital - p. ej.: audio, visual, audiovisual, multimedia y otros-.

Las TIC proporcionan una opción vasta de herramientas didácticas para la enseñanza/aprendizaje que buscan motivar al estudiante a aprender y fortalecer sus competencias para un aprendizaje autónomo, de tal manera que esté preparado para acceder a diversos entornos educativos mediados por las nuevas tecnologías (Escobar y Buitrago, 2017). En este sentido, las TIC condicionan a que los profesionales de la enseñanza no se perpetúen en el modelo tradicional de formación del siglo XX, más aún cuando todos los ámbitos de nuestra vida están experimentándose a través de una pantalla —dispositivos móviles, computadores, pizarras digitales y otros dispositivos de uso cotidiano conectados a través del internet- (Durán y Durán, 2017). Todo esto implica un cambio de actitud para adaptarse a nuevas formas de enseñanza/ aprendizaje en una sociedad cada día más digitalizada (Durán y Durán, 2017).
En cuanto a las herramientas de autor en la práctica docente, ¿qué valor pueden llegar a tener si los docentes no saben utilizarlas como herramientas educativas para integrarlas en los procesos de enseñanza/aprendizaje?, ¿qué competencias digitales exigen al docente para poder utilizarlas?, ¿los contenidos web generados por estas herramientas pueden ser consumidos por una gran mayoría de estudiantes con diferentes necesidades y preferencias de aprendizaje?

Para dar atención a las indagaciones antes mencionadas, un docente tiene que hacer votos por una capacitación constante en aspectos tecnológicos para estar actualizado con la era digital; de este modo, podría integrar nuevas didácticas en el aula mediadas por las TIC. En este sentido, resulta relevante un estudio como el descrito en este artículo, que buscó indagar el nivel de experticia o habilidad de los docentes en determinadas competencias digitales, así como también identificar aquellas herramientas de autor que soportan la producción de contenido web en el contexto de la práctica docente en Colombia. Este estudio es la base para un análisis ulterior que permitirá determinar en qué medida el contenido web generado por estas herramientas y las mismas herramientas son accesibles.

Este documento está estructurado de la siguiente manera: en primer lugar, se presenta la metodología adoptada para el propósito de esta investigación, que incluye tres etapas claramente definidas: a) revisión bibliográfica para 
la búsqueda, organización y análisis de documentación en diferentes fuentes de consulta; brindando información de las herramientas de autor más referenciadas en la docencia, b) recolección de datos e información primaria, mediante la aplicación de una encuesta a una muestra de docentes en el departamento de Antioquia y c) tabulación de los resultados de la encuesta. Segundo, se analizan los resultados de la encuesta y se presenta la información obtenida. Finalmente, con base en la indagación realizada, se presentan las conclusiones.

\section{Metodología}

Los procesos culturales son dinámicos y vulnerables a los cambios sociales, a la globalización, a la acelerada urbanización, a la desvalorización social, a la pérdida de referentes culturales, al desuso, a la pérdida de autoestima de las comunidades, a la ruptura generacional y a la excesiva comercialización. Todos estos factores pueden resultar en impactos no deseados del patrimonio cultural nacional y, en consecuencia, se deben crear planes especiales de salvaguardarlo.

La metodología descrita a continuación permitió identificar aquellas herramientas de autor más usadas en la práctica docente; así mismo, se realizó una caracterización rigurosa de la muestra de docentes colombianos que participaron en el estudio, lo cual ayudó a contextualizar muy bien los resultados de la investigación. En particular, se analizó la información demográfica de los participantes como ciertas competencias TIC consideradas relevantes para el estudio. Para este propósito, la metodología seguida fue la siguiente:

\subsection{Revisión bibliográfica}

De manera preliminar y con base en la literatura académica que aportan al contexto de este estudio, se buscó identificar aquellas herramientas de autor que apoyan la gestión y práctica docente en Colombia.

\subsection{Recolección de datos e información}

Posteriormente, y con base en las herramientas de autor identificadas en la revisión bibliográfica, se elaboró una encuesta en línea que fue aplicada a una muestra de docentes colombianos ubicados en el departamento de Antioquia.

\subsection{Tabulación de los resultados de la encuesta}

Finalmente, se tabularon los datos obtenidos en la encuesta para su ulterior análisis de resultados. En los siguientes capítulos se detallarán cada una de las etapas llevadas a cabo en profundidad.

\section{Desarrollo del contenido}

\subsection{Revisión bibliográfica}

Para la identificación de las aplicaciones que apoyan la gestión y práctica docente en Colombia, se usó la metodología de 
revisión bibliográfica propuesta por Gómez-Luna, Navas, Aponte-Mayor y Betancourt (2014), de modo que se ejecutaron las siguientes fases:

\subsubsection{Planificación de la búsqueda.}

Se planificó una búsqueda bibliográfica, definiéndose como tema de búsqueda: "herramientas de autor que apoyan la gestión y práctica docente». Además, se formularon factores de búsqueda -desagregación del tema-, preguntas orientadoras de búsqueda ( $\mathrm{KIQ}$, key information question) y palabras clave de búsqueda -keywords-; orientando la indagación, tal y como se muestra en la tabla 1.

Tabla 1. Planificación de la búsqueda bibliográfica

\begin{tabular}{|c|c|c|c|}
\hline Factores & KIQ & Keywords & $\begin{array}{c}\text { Alcance / } \\
\text { limitaciones }\end{array}$ \\
\hline $\begin{array}{c}\text { Plataformas o } \\
\text { entornos virtuales } \\
\text { de aprendizaje. }\end{array}$ & $\begin{array}{c}\text { ¿Qué } \\
\text { plataformas } \\
\text { o entornos } \\
\text { virtuales de } \\
\text { aprendizaje son } \\
\text { usados en la } \\
\text { educación? }\end{array}$ & $\begin{array}{l}\text { EVA, EVEA, } \\
\text { LMS, } \\
\text { plataforma } \\
\text { virtual, } \\
\text { enseñanza, } \\
\text { aprendizaje. }\end{array}$ & $\begin{array}{l}\text { Colombia, otras } \\
\text { referencias } \\
\text { internacionales, } \\
\text { fecha de } \\
\text { publicación: } \\
\text { desde el } 2013 \\
\text { hasta el } 2017 \\
\text { inclusive. }\end{array}$ \\
\hline $\begin{array}{c}\text { Herramientas TIC } \\
\text { para creación de } \\
\text { contenido web } \\
\text { con propósito } \\
\text { educativo. }\end{array}$ & $\begin{array}{c}\text { ¿Qué } \\
\text { herramientas de } \\
\text { autor apoyan } \\
\text { los procesos } \\
\text { de enseñanza y } \\
\text { aprendizaje? }\end{array}$ & $\begin{array}{c}\text { Herramientas } \\
\text { de autor, TIC, } \\
\text { enseñanza, } \\
\text { aprendizaje, } \\
\text { docente, Web } \\
\text { 2.0, educación }\end{array}$ & $\begin{array}{l}\text { Colombia, otras } \\
\text { referencias } \\
\text { internacionales, } \\
\text { fecha de } \\
\text { publicación: } \\
\text { desde el } 2013 \\
\text { hasta el } 2017 \\
\text { inclusive. }\end{array}$ \\
\hline
\end{tabular}

Fuente. Adaptación realizada de la plantilla de Vigilancia Tecnológica del Centro de Investigación Para el Desarrollo y la Innovación-Universidad Pontificia Bolivariana (Centro de Investigación para el Desarrollo y la Innovación, CIDI, 2017).

\subsubsection{Búsqueda de información.}

Una vez definidas las preguntas orientadoras de la búsqueda bibliográfica, se consultaron las siguientes bases de datos académicas y repositorios digitales: Google Scholar, EBSCOHost, Scopus, Repositorio Digital de la Universidad Nacional, sede Medellín: bdigital y Repositorio Digital de la Universidad de los Andes.

\subsubsection{Selección de la información.}

La búsqueda dio como resultado 234 documentos, de entre estos se identificó a 20 publicaciones con información significativa para este estudio. El procedimiento aplicado para la selección de las referencias bibliográficas consistió en:

a) La lectura del tema y resumen, como primer criterio de selectividad.

b) La descarga del archivo digital del documento seleccionado en formato PDF.

c) La aplicación de la técnica de scanning -lectura rápidade las secciones de: introducción, presentación o análisis de resultados, conclusiones y recomendaciones, de manera que se estableciera un segundo criterio de selección del documento.

Según los dos criterios antes mencionados, se decidió incluir o excluir al documento de la revisión bibliográfica. Si el documento era seleccionado, se registraba la información requerida en una bitácora de búsqueda -instrumento construido para tal propósito-. 


\subsubsection{Organización de la información.}

Se usó el software de gestión de referencias bibliográficas Mendeley para organizar y almacenar los 20 documentos filtrados en la fase de búsqueda de la información.

\subsubsection{Resultados.}

En resumen, la Tabla 2 sintetiza el resultado de la revisión bibliográfica.

Tabla 2. Resultados de la revisión bibliográfica

\begin{tabular}{|c|c|c|}
\hline Autor(es) & LMS identificado(s) & $\begin{array}{c}\text { Herramienta(s) de } \\
\text { autor identificada(s) }\end{array}$ \\
\hline Arnaldos et al. (2015) & Moodle, Sakai. & - \\
\hline Aguilar y Ayala (2014) & $\begin{array}{c}\text { Moodle, Sakai y } \\
\text { Dokeos. }\end{array}$ & $\begin{array}{c}\text { Ardora, Constructor, } \\
\text { Cuadernia, EdiLim, } \\
\text { eXeLearning, Hot } \\
\text { Potatoes y JClic. }\end{array}$ \\
\hline Arangure (2013) & Moodle & - \\
\hline Barrionuevo, Hidalgo, & - & $\begin{array}{c}\text { Ardora, eXeLearning, } \\
\text { Cuadernia, Lim y Hot } \\
\text { Potatoes }\end{array}$ \\
\hline Bucheli y Ortiz (2017) & Moodle & - \\
\hline $\begin{array}{c}\text { Cuetía y Sanjuán } \\
\text { (2017) }\end{array}$ & Moodle & - \\
\hline $\begin{array}{c}\text { Echeverria y Cobos } \\
\text { (2015) }\end{array}$ & Moodle & Edmodo, blogs y wikis \\
\hline González (2015) & - & - \\
\hline
\end{tabular}

\begin{tabular}{|c|c|c|}
\hline Henao (2013) & - & $\begin{array}{c}\text { eXeLearning, weblogs } \\
\text { y wikis }\end{array}$ \\
\hline Hernández (2013) & - & Erudito y Hot Potatoes \\
\hline Jara (2017) & Moodle & - \\
\hline Jiménez (2014) & Moodle & - \\
\hline Kasim y Khalid (2016) & $\begin{array}{c}\text { Moodle, Sakai y } \\
\text { ATutor. }\end{array}$ & - \\
\hline Marín (2014) & Moodle & Edmodo \\
\hline Ortiz (2017) & - & Blogger y WordPress \\
\hline Ramírez (2016) & Moodle & $\begin{array}{c}\text { Edmodo, EducaPlay y } \\
\text { Hot Potatoes }\end{array}$ \\
\hline $\begin{array}{c}\text { Tárraga y Colomer } \\
\text { (2013) }\end{array}$ & - & $\begin{array}{c}\text { Ardora, Constructor y } \\
\text { JClic }\end{array}$ \\
\hline $\begin{array}{c}\text { Vargas, Villamil, y } \\
\text { Tarazona (2017) }\end{array}$ & $\begin{array}{c}\text { ATutor, Chamilo, } \\
\text { Claroline y Moodle. }\end{array}$ & - \\
\hline Violini y Sanz (2016) & - & $\begin{array}{c}\text { Ardora, eXeLearning } \\
\text { y Xerte. }\end{array}$ \\
\hline
\end{tabular}

Fuente. Elaboración propia

\subsection{Recolección de datos e información primaria}

Con el propósito de obtener datos primarios sobre el uso de herramientas de autor por los docentes en los espacios de educación en Colombia, y como instrumento de investigación, se creó una encuesta que fue completada por 22 docentes colombianos. En síntesis, para la aplicación de la encuesta se realizaron las siguientes acciones: 


\subsubsection{Diseño del cuestionario.}

Se diseñó un cuestionario para ser aplicado en una herramienta de recolección de datos en línea; teniendo como referencia aquellas herramientas de autor identificadas en la revisión bibliográfica.

Este cuestionario tuvo como finalidad analizar el uso de las TIC por el profesorado. Sus propósitos fueron: a) detectar el conocimiento o habilidades en el uso de las TIC; y b) identificar las herramientas de autor más conocidas o usadas para el diseño de recursos educativos. Por otra parte, este instrumento de recolección de datos se compuso de tres secciones: a) información general, para obtener datos de las variables sociales, b) competencias TIC, para indagar sobre el conocimiento o habilidades básicas en el uso del computador e internet $y$, de manera fundamental para esta investigación, c) el uso de las TIC en la práctica docente, donde se hace énfasis en la identificación de las herramientas de autor que son utilizadas por los docentes para la creación de contenido web con propósito educativo y con características accesibles.

\subsubsection{Publicación del cuestionario en línea.}

Se creó y validó una cuenta de usuario en la plataforma Survey Monkey, una herramienta en línea para encuestas, y allí se diseñó y se publicó el cuestionario.

\subsubsection{Selección de la muestra.}

La muestra objeto de estudio de esta investigación fueron 69 docentes del programa de becas de la Secretaría de
Educación de Antioquia (SEDUCA). Para este propósito, se elaboró un listado de este grupo objetivo, al cual se aplicará la encuesta en línea. Se requirió la siguiente información: nombres del encuestado, institución de educación en la que labora y correo electrónico -ningún dato sensible de los encuestados será publicado, de modo que se garantice la reserva y anonimato de la encuesta-.

\subsubsection{Aplicación del instrumento de recolección de datos.}

Se solicitó el diligenciamiento de la encuesta en línea vía correo electrónico al grupo objetivo de estudio: 69 docentes. De esta muestra, 22 encuestados respondieron al llamado para participar de esta investigación. Todos completaron la encuesta en su totalidad.

\subsection{Tabulación de los resultados de la encuesta}

La información obtenida de cada pregunta fue tabulada en una hoja de cálculo en Microsoft Excel. El procedimiento para el tratamiento y tabulación de respuestas múltiples en la investigación fue tomado de Serrano (2013).

\section{Resultados}

Con base en el tratamiento y análisis de la información obtenida, se describen los resultados a continuación. La Tabla 3 presenta los datos demográficos básicos recopilados. Alrededor del $54 \%$ de los encuestados eran del sexo femenino, y alrededor del 32 \% se ubicó en el ulterior grupo 
de edad más joven (37-41 años). La población encuestada formó parte del programa de becas de posgrado de la Secretaría de Educación de Antioquia (SEDUCA); por ello, alrededor del $96 \%$ tiene un título de maestría. Finalmente, ninguno considera tener alguna limitación para el acceso a la tecnología.

Tabla 3. Distribución de las variables sociales

\begin{tabular}{|c|c|c|}
\hline Variables & Frecuencia & Porcentaje \\
\hline \multicolumn{3}{|c|}{ Edad } \\
\hline $18-36$ & 2 & $9,09 \%$ \\
\hline $37-41$ & 7 & $31,82 \%$ \\
\hline $42-46$ & 4 & $18,18 \%$ \\
\hline $47-51$ & 6 & $27,27 \%$ \\
\hline $52-56$ & 3 & $13,64 \%$ \\
\hline \multicolumn{3}{|c|}{ Género } \\
\hline Masculino & 10 & $45,45 \%$ \\
\hline Femenino & 12 & $54,55 \%$ \\
\hline \multicolumn{3}{|c|}{ Título académico } \\
\hline Normal superior & 0 & $0,00 \%$ \\
\hline $\begin{array}{c}\text { Licenciatura o nivel } \\
\text { equivalente (pregrado) }\end{array}$ & 1 & $4,55 \%$ \\
\hline $\begin{array}{c}\text { Especialización } \\
\text { (posgrado) }\end{array}$ & 0 & $0,00 \%$ \\
\hline Maestría (posgrado) & 21 & $95,45 \%$ \\
\hline
\end{tabular}

\begin{tabular}{|c|c|c|}
\hline \multicolumn{2}{|c|}{ Limitación para el acceso a la tecnología } \\
\hline $\begin{array}{c}\text { Limitación } \\
\text { permanente }\end{array}$ & 0 & $0,00 \%$ \\
\hline Limitación temporal & 0 & $0,00 \%$ \\
\hline Ninguna & 22 & $100,00 \%$ \\
\hline
\end{tabular}

Nota: Las sumatorias parciales de las frecuencias en cada ítem son 22 en total, el número de encuestados.

Fuente. Elaboración propia.

La Tabla 4 muestra los resultados de la encuesta en cuanto al conocimiento en el uso del computador y el internet. En forma general, la mayoría de los encuestados indicaron tener un nivel intermedio de experticia, tanto para el manejo de computadores $(68,18 \%)$ como para navegar en internet $(72,73 \%)$. Al analizar el uso del internet, alrededor del $91 \%$ dijo usarlo hace más de 5 años y alrededor del $41 \%$ lo emplea por más de 15 horas a la semana.

Tabla 4. Experticia en el uso del computador e internet

\begin{tabular}{|c|c|c|}
\hline Variables & Frecuencia & Porcentaje \\
\hline Nivel de experticia usando computadores \\
\hline Principiante & 0 & $0,00 \%$ \\
\hline Intermedio & 15 & $68,18 \%$ \\
\hline Experto & 7 & $31,82 \%$ \\
\hline \multicolumn{3}{|c|}{ Nivel de experticia usando internet } \\
\hline Principiante & 0 & $0,00 \%$ \\
\hline Intermedio & 16 & $72,73 \%$ \\
\hline Experto & 6 & $27,27 \%$ \\
\hline
\end{tabular}




\begin{tabular}{|c|c|c|}
\hline \multicolumn{3}{|c|}{ Tiempo de experiencia usando internet } \\
\hline Menos de un año & 0 & $0,00 \%$ \\
\hline 1 - 2 años & 0 & $0,00 \%$ \\
\hline 3 - 5 años & 2 & $9,09 \%$ \\
\hline Más de 5 años & 20 & $90,91 \%$ \\
\hline \multicolumn{2}{|c|}{ Horas de uso de internet por semana } \\
\hline $\begin{array}{c}\text { Al menos una hora a la } \\
\text { semana }\end{array}$ & 0 & $0,00 \%$ \\
\hline $\begin{array}{c}\text { Entre 2 y } 5 \text { horas a la } \\
\text { semana }\end{array}$ & 6 & $27,27 \%$ \\
\hline $\begin{array}{c}\text { Entre 6 y } 15 \text { horas a la } \\
\text { semana }\end{array}$ & 7 & $31,82 \%$ \\
\hline $\begin{array}{c}\text { Más de 15 horas a la } \\
\text { semana }\end{array}$ & 9 & $40,91 \%$ \\
\hline Nata: Las sumatio para & \\
\hline
\end{tabular}

Nota: Las sumatorias parciales de las frecuencias en cada ítem son 22 en total, el número de encuestados.

Fuente. Elaboración propia.

En la tabla 5 la muestra reflejó el bajo nivel de conocimiento o habilidad para la instalación de software en el computador: el 18,18 \% -ninguna-y el 13,64 \% -poco-. Por otra parte, la gran mayoría indicó que está capacitado para navegar en internet y localizar información -alrededor del $68 \%$ lo maneja bien y alrededor del 32 \% muy bien-, así como para usar el correo electrónico -alrededor del $18 \%$ lo maneja bien y alrededor del 82 \% muy bien-.
Tabla 5. Competencias informáticas software e internet

\begin{tabular}{|c|c|c|}
\hline Variables & Frecuencia & Porcentaje \\
\hline \multicolumn{3}{|c|}{ Instala software en el computador } \\
\hline Nada & 4 & $18,18 \%$ \\
\hline Poco & 3 & $13,64 \%$ \\
\hline Regular & 2 & $9,09 \%$ \\
\hline Bien & 5 & $22,73 \%$ \\
\hline Muy bien & 8 & $36,36 \%$ \\
\hline
\end{tabular}

Navega en internet mediante un buscador para localizar información (ejemplo: documentos, videos, canciones)

\begin{tabular}{|c|c|c|}
\hline Nada & 0 & $0,00 \%$ \\
\hline Poco & 0 & $0,00 \%$ \\
\hline Regular & 0 & $0,00 \%$ \\
\hline Bien & 7 & $31,82 \%$ \\
\hline Muy bien & 15 & $68,18 \%$ \\
\hline \multicolumn{2}{|c|}{ Utiliza el correo electrónico } \\
\hline (ejemplo: enviar/recibir mensajes, adjuntar archivos) \\
\hline Nada & 0 & $0,00 \%$ \\
\hline Poco & 0 & $0,00 \%$ \\
\hline Regular & 0 & $0,00 \%$ \\
\hline Bien & 4 & $18,18 \%$ \\
\hline Muy bien & 18 & $81,82 \%$ \\
\hline
\end{tabular}

Nota: Las sumatorias parciales de las frecuencias en cada ítem son 22 en total, el número de encuestados.

Fuente. Elaboración propia.

En la Tabla 6 se analiza que la mayoría de docentes indicaron manejar muy bien competencias que son básicas para la creación de contenidos en procesadores o editores 
de texto, como son: trabajar con archivos y carpetas en un sistema operativo $(77,27 \%)$, respaldar información en diferentes soportes $(68,18 \%)$ o crear y editar documentos de texto $(72,73 \%)$.

Tabla 6. Competencias básicas en editores de texto

\begin{tabular}{|c|c|c|}
\hline Variables & Frecuencia & Porcentaje \\
\hline \multicolumn{3}{|c|}{$\begin{array}{c}\text { Trabaja con archivos y carpetas en el sistema operativo } \\
\text { (crear, copiar, mover y eliminar) }\end{array}$} \\
\hline Nada & 0 & $0.00 \%$ \\
\hline Poco & 0 & $0,00 \%$ \\
\hline Regular & 1 & $4,55 \%$ \\
\hline Bien & 4 & $18,18 \%$ \\
\hline Muy bien & 17 & $77,27 \%$ \\
\hline \multicolumn{3}{|c|}{$\begin{array}{c}\text { Guarda y recupera información en diferentes soportes } \\
\text { (ejemplos: CD/DVD, memoria USB, almacenamiento en la nube) }\end{array}$} \\
\hline Nada & 0 & $0,00 \%$ \\
\hline Poco & 0 & $0,00 \%$ \\
\hline Regular & 1 & $4,55 \%$ \\
\hline Bien & 6 & $27,27 \%$ \\
\hline Muy bien & 15 & $68,18 \%$ \\
\hline \multicolumn{3}{|c|}{ Crea y edita documentos de texto } \\
\hline Nada & 0 & $0,00 \%$ \\
\hline Poco & 0 & $0,00 \%$ \\
\hline Regular & 2 & $9,09 \%$ \\
\hline Bien & 4 & $18,18 \%$ \\
\hline Muy bien & 16 & $72,73 \%$ \\
\hline
\end{tabular}

Nota: Las sumatorias parciales de las frecuencias en cada ítem son 22 en total, el número de encuestados.

Fuente. Elaboración propia.
Al indagar sobre el uso de aplicaciones para la creación de contenido multimedia, los docentes indicaron tener conocimiento o habilidades para usar software que permiten brindar información en múltiples medios y en distintas formas de presentación; así, se obtuvo que alrededor del $55 \%$ indican manejar muy bien dichas herramientas, como se observa en la tabla 7.

Tabla 7. Competencia digital creación de contenido multimedia

\begin{tabular}{|c|c|c|}
\hline Variables & Frecuencia & Porcentaje \\
\hline \multicolumn{2}{|c|}{ Utiliza aplicaciones para la creación de contenido multimedia: } \\
sonido, video, imágenes. \\
\hline Nada & 0 & $0,00 \%$ \\
\hline Poco & 0 & $0,00 \%$ \\
\hline Regular & 4 & $18,18 \%$ \\
\hline Bien & 6 & $27,27 \%$ \\
\hline Muy bien & 12 & $54,55 \%$ \\
\hline Total & 22 & $100,00 \%$ \\
\hline
\end{tabular}

Fuente. Elaboración propia.

En la tabla 8 se observan los datos obtenidos en referencia con el uso de otras formas de comunicación y socialización; se puede anotar que el 9,09 \% maneja poco tanto los espacios de participación (foros, blogs o wikis) como el uso de aplicaciones para videoconferencias. Así mismo, un porcentaje mayoritario de los encuestados, indica tener capacidad para el uso de redes sociales como Facebook, Instagram o YouTube: 40,91 \% —bien- y 50,00 \% —muy bien-. 
Tabla 8. Competencias informáticas de otras formas de comunicación

\begin{tabular}{|c|c|c|}
\hline Variables & Frecuencia & Porcentaje \\
\hline \multicolumn{2}{|c|}{ Utiliza otras formas de comunicación como foros de discusión, } \\
blogs o wikis \\
\hline Nada & 0 & $0,00 \%$ \\
\hline Poco & 2 & $9,09 \%$ \\
\hline Regular & 2 & $9,09 \%$ \\
\hline Bien & 7 & $31,82 \%$ \\
\hline Muy bien & 11 & $50,00 \%$ \\
\hline
\end{tabular}

Utiliza aplicaciones para videoconferencias como Skype o Hangouts

\begin{tabular}{|c|c|c|}
\hline Nada & 0 & $0,00 \%$ \\
\hline Poco & 2 & $9,09 \%$ \\
\hline Regular & 3 & $13,64 \%$ \\
\hline Bien & 5 & $22,73 \%$ \\
\hline Muy bien & 12 & $54,55 \%$ \\
\hline
\end{tabular}

Utiliza aplicaciones para redes sociales como Facebook®, Instagram $\AA_{\text {, YouTube }}{ }^{\circ}$

\begin{tabular}{|c|c|c|}
\hline Nada & 0 & $0,00 \%$ \\
\hline Poco & 0 & $0,00 \%$ \\
\hline Regular & 2 & $9,09 \%$ \\
\hline Bien & 9 & $40,91 \%$ \\
\hline Muy bien & 11 & $50,00 \%$ \\
\hline
\end{tabular}

Nota: Las sumatorias parciales de las frecuencias en cada ítem son 22 en total, el número de encuestados.

Fuente. Elaboración propia.

La tabla 9 muestra que todos los encuestados han recibido al menos alguna vez un curso de capacitación en el uso de las TIC en los procesos educativos, se resalta que la mayoría lo hizo recientemente, por lo menos uno en el último año $(68,18 \%)$ y dos o más en el último año (22,73\%). Además, todos los encuestados (100,00 \%) indicaron que es muy importante integrar el uso de las TIC en las aulas. Todo esto resalta la necesidad de mejorar y fortalecer la calidad de la propuesta educativa y su articulación con las nuevas tecnologías.

Tabla 9. Uso de las TIC en los procesos educativos

\begin{tabular}{|c|c|c|}
\hline Variables & Frecuencia & Porcentaje \\
\hline \multicolumn{3}{|c|}{ Capacitación en el uso adecuado de las TIC en los procesos educativos } \\
\hline $\begin{array}{l}\text { Por lo menos uno en } \\
\text { el último año }\end{array}$ & 15 & $68,18 \%$ \\
\hline $\begin{array}{c}\text { Dos o más en el } \\
\text { último año }\end{array}$ & 5 & $22,73 \%$ \\
\hline $\begin{array}{l}\text { Por lo menos uno } \\
\text { hace más de un año }\end{array}$ & 2 & $9,09 \%$ \\
\hline Ninguno & 0 & $0,00 \%$ \\
\hline No sabe/no conoce & 0 & $0,00 \%$ \\
\hline \multicolumn{3}{|c|}{ Importancia uso de las TIC en las aulas } \\
\hline Muy importante & 22 & $100,00 \%$ \\
\hline Importante & 0 & $0,00 \%$ \\
\hline $\begin{array}{l}\text { Moderadamente } \\
\text { importante }\end{array}$ & 0 & $0,00 \%$ \\
\hline De poca importancia & 0 & $0,00 \%$ \\
\hline Sin importancia & 0 & $0,00 \%$ \\
\hline
\end{tabular}

Fuente. Elaboración propia. 
De acuerdo con la tabla 10, el $45,45 \%$ de los encuestados señaló haber diseñado ocasionalmente recursos mediados por TIC. Así mismo, un 45,45 \% indicó que ocasionalmente considera la accesibilidad en diseño de dichos recursos educativos. Apenas el 4,55 \% dice no haber diseñado nunca recursos digitales.

Tabla 10. Diseño de recursos educativos mediados por TIC

\begin{tabular}{|c|c|c|}
\hline Variables & Frecuencia & Porcentaje \\
\hline \multicolumn{3}{|c|}{ Diseño recursos educativos mediados por TIC } \\
\hline Muy frecuentemente & 5 & $22,73 \%$ \\
\hline Frecuentemente & 6 & $27,27 \%$ \\
\hline Ocasionalmente & 10 & $45,45 \%$ \\
\hline Raramente & 0 & $0,00 \%$ \\
\hline Nunca & 1 & $4,55 \%$ \\
\hline No sabe/no conoce & 0 & $0,00 \%$ \\
\hline \multicolumn{3}{|c|}{ Accesibilidad diseño recursos educativos } \\
\hline Muy frecuentemente & 2 & $9,09 \%$ \\
\hline Frecuentemente & 7 & $31,82 \%$ \\
\hline Ocasionalmente & 10 & $45,45 \%$ \\
\hline Raramente & 1 & $4,55 \%$ \\
\hline Nunca & 1 & $4,55 \%$ \\
\hline $\begin{array}{c}\text { No diseña recursos } \\
\text { educativos }\end{array}$ & 1 & $4,55 \%$ \\
\hline No sabe/no conoce & 0 & $0,00 \%$ \\
\hline
\end{tabular}

Fuente. Elaboración propia.
Por otra parte, la tabla 11 muestra que alrededor del $36,00 \%$ integra frecuentemente recursos educativos mediados por TIC en los centros educativos y alrededor del $41,00 \%$ indicó hacerlo ocasionalmente. En un porcentaje menor, alrededor del 9,00 \% señalaron que usan raramente estas herramientas como recursos didácticos.

Tabla 11. Uso de herramientas de autor en la práctica docente

\begin{tabular}{|c|c|c|}
\hline $\begin{array}{c}\text { Uso de herramientas TIC en la } \\
\text { práctica docente }\end{array}$ & Frecuencia & Porcentaje \\
\hline Muy frecuentemente & 3 & $13,64 \%$ \\
\hline Frecuentemente & 8 & $36,36 \%$ \\
\hline Ocasionalmente & 9 & $40,91 \%$ \\
\hline Raramente & 2 & $9,09 \%$ \\
\hline Nunca & 0 & $0,00 \%$ \\
\hline Total & 22 & $100,00 \%$ \\
\hline
\end{tabular}

Fuente. Elaboración propia.

La tabla 12 muestra que la mayoría ha usado una plataforma o entorno virtual de aprendizaje: muy frecuentemente $(18,18 \%)$, frecuentemente $(45,45 \%)$ y ocasionalmente $(31,82 \%)$.

Tabla 12. Frecuencia de uso entorno virtual de aprendizaje

\begin{tabular}{|c|c|c|}
\hline $\begin{array}{c}\text { Uso de una plataforma o entorno } \\
\text { virtual de aprendizaje }\end{array}$ & Frecuencia & Porcentaje \\
\hline Muy frecuentemente & 4 & $18,18 \%$ \\
\hline Frecuentemente & 10 & $45,45 \%$ \\
\hline Ocasionalmente & 7 & $31,82 \%$ \\
\hline
\end{tabular}




\begin{tabular}{|c|c|c|}
\hline Raramente & 1 & $4,55 \%$ \\
\hline Nunca & 0 & $0,00 \%$ \\
\hline No sabe/no conoce & 0 & $0,00 \%$ \\
\hline Total & 22 & $100,00 \%$ \\
\hline
\end{tabular}

Fuente. Elaboración propia.

Los datos en la tabla 13 reflejan que las plataformas o entornos de aprendizaje que los encuestados han oído mencionar con mayor frecuencia son: Moodle (37,74 \%) y ATutor (35,85\%). Además, estas plataformas han sido señaladas como las más utilizadas: Moodle $(44,44 \%)$ y ATutor (37,78\%).

Tabla 13. Plataformas o entornos virtuales de aprendizaje

\begin{tabular}{|c|c|c|}
\hline Variables & Frecuencia (f) & Porcentaje (f/Total) \\
\hline \multicolumn{2}{|c|}{ Plataforma o entorno virtual de aprendizaje que ha oído mencionar } \\
\hline Moodle & 20 & $37,74 \%$ \\
\hline ATutor & 19 & $35,85 \%$ \\
\hline Sakai & 3 & $5,66 \%$ \\
\hline Chamilo & 3 & $5,66 \%$ \\
\hline Ninguna / no conoce & 0 & $0,00 \%$ \\
\hline Otro & 8 & $15,09 \%$ \\
\hline Total & 53 & $100,00 \%$ \\
\hline Plataforma o entorno virtual de aprendizaje que ha utilizado \\
\hline Moodle & 20 & $44,44 \%$ \\
\hline ATutor & 17 & $37,78 \%$ \\
\hline Sakai & 1 & $2,22 \%$ \\
\hline
\end{tabular}

\begin{tabular}{|c|c|c|}
\hline Chamilo & 0 & $0,00 \%$ \\
\hline Ninguna / no conoce & 0 & $0,00 \%$ \\
\hline Otro & 7 & $15,56 \%$ \\
\hline Total & 45 & $100,00 \%$ \\
\hline
\end{tabular}

Nota: Número de encuestas: 22. El procedimiento para el tratamiento y tabulación de respuestas múltiples en la investigación fue tomado de Serrano (2013).

Fuente. Elaboración propia.

De otra parte, también se indagó aquellas herramientas de autor que no son consideradas como entornos o plataformas virtuales de aprendizaje. La tabla 14 muestra que las aplicaciones que más han oído mencionar son: EdModo $(20,93 \%)$, Blogger $(25,58 \%)$, JClic $(13,95 \%)$ y HotPotatoes $(10,47 \%)$. Por otra parte, alrededor del $34,00 \%$ seleccionó a Blogger como la aplicación más utilizada, seguida de EdModo $(18,03 \%)$, JClic $(18,03 \%)$ y HotPotatoes $(11,48 \%)$.

Tabla 14. Herramientas de autor

\begin{tabular}{|c|c|c|}
\hline Variables & Frecuencia $(\mathbf{f})$ & Porcentaje (f/Total) \\
\hline \multicolumn{2}{|c|}{ Herramientas de autor que ha oído mencionar } \\
\hline Hot Potatoes & 9 & $10,47 \%$ \\
\hline JClic & 12 & $13,95 \%$ \\
\hline Ardora & 1 & $1,16 \%$ \\
\hline eXeLearning & 9 & $10,47 \%$ \\
\hline EdModo & 18 & $20,93 \%$ \\
\hline WordPress & 12 & $13,95 \%$ \\
\hline Blogger & 22 & $25,58 \%$ \\
\hline
\end{tabular}




\begin{tabular}{|c|c|c|}
\hline Ninguna/no conoce & 0 & $0,00 \%$ \\
\hline Otro & 3 & $3,49 \%$ \\
\hline Total & 86 & $100,00 \%$ \\
\hline \multicolumn{2}{|c|}{ Herramientas de autor que ha utilizado } \\
\hline Hot Potatoes & 7 & $11,48 \%$ \\
\hline JClic & 11 & $18,03 \%$ \\
\hline Ardora & 0 & $0,00 \%$ \\
\hline eXeLearning & 5 & $8,20 \%$ \\
\hline EdModo & 11 & $18,03 \%$ \\
\hline WordPress & 3 & $4,92 \%$ \\
\hline Blogger & 21 & $34,43 \%$ \\
\hline Ninguna / No conoce & 0 & $0,00 \%$ \\
\hline Otro (especifique) & 3 & $4,92 \%$ \\
\hline Total & 61 & $100,00 \%$ \\
\hline
\end{tabular}

Nota: Número de encuestas: 22. El procedimiento para el tratamiento y tabulación de respuestas múltiples en la investigación fue tomado de: Serrano (2013).

Fuente. Elaboración propia.

\section{Conclusiones}

En este estudio se lograron identificar aquellas herramientas de autor que se consideran como las más conocidas o usadas por los docentes colombianos, en particular los antioqueños. Si bien los docentes manifestaron tener competencias digitales para el uso del computador e internet, así como estar de acuerdo con la importancia del uso de las TIC para apuntalar la transformación, innovación y mejorara de los métodos tradicionales de enseñanza y aprendizaje, esto no ha sido adoptado por la mayoría de educadores encuestados como didáctica de uso frecuente en las aulas. Esto se denota con la frecuencia ocasional en la que diseñan recursos didácticos mediados por TIC como herramienta en su práctica docente.

Por otro lado, un grupo significativo de docentes no consideran muy frecuentemente la accesibilidad para el diseño de recursos educativos mediados por TIC, por ello, se hace necesaria la sensibilización y la adquisición de competencias digitales para la creación de material educativo mediado por TIC que consideren los lineamientos de accesibilidad web. Así, se incluye a una mayoría de alumnado con diferentes necesidades y estilos de aprendizaje.

Como trabajo futuro, este estudio será la base para analizar más en detalle la accesibilidad de las herramientas de autor identificadas, así como del contenido web que generan. 


\section{Referencias}

Centro de Investigación para el Desarrollo y la Innovación (CIDI). (2017). VT e IC Herramienta estratégica para la competitividad [Material de clase]. Medellín, Colombia: Universidad Pontificia Bolivariana.

Crosetti, B. (2006). Herramientas para la creación, distribución y gestión de cursos a través de Internet. Edutec. Revista Electrónica de Tecnología Educativa, 26(12), 1-7. DOI: https:// doi.org/10.21556/edutec.2000.12.556

Durán, J. F.; Durán, I. (2017). TIC actualizadas para una nueva docencia universitaria. Madrid: Mc Graw Hill Interamericana S.L.

Escobar, D.; Buitrago, H. (2017). La aplicación de las TIC en el aula de clase, opciones de herramientas didácticas para fortalecer las prácticas de enseñanza. Ponencia presentada en las IV Jornadas de TIC e Innovación En El Aula. Universidad Nacional de La Plata, Argentina, noviembre.

Gómez-Luna, E.; Navas, D.; Aponte-Mayor, G.; Betancourt-Buitrago, L. (2014). Literature review methodology for scientific and information management, through its structuring and systematization. Dyna, 81(184), 158-163. DOI: https://doi. org/10.15446/dyna.v81n184.37066

Serrano J. (2013). Respuestas múltiples en la investigación educativa: Codificación, tabulación y análisis. Revista de Investigación Educativa, 31(2), 361-374. DOI: https://doi.org/10.6018/ rie.31.2.164111 